\title{
A short proof of a partition relation for triples
}

\author{
Albin L. Jones \\ Department of Mathematics \\ Kenyon College \\ Gambier, OH 43022, USA \\ jones@kenyon.edu \\ http://math.kenyon.edu/ jones/
}

Submitted: September 3, 1999; Accepted: March 11, 2000

\begin{abstract}
We provide a much shorter proof of the following partition theorem of P. Erdős and R. Rado: If $X$ is an uncountable linear order into which neither $\omega_{1}$ nor $\omega_{1}^{*}$ embeds, then $X \rightarrow(\alpha, 4)^{3}$ for every ordinal $\alpha<\omega+\omega$. We also provide two counterexamples to possible generalizations of this theorem, one of which answers a question of E. C. Milner and K. Prikry.
\end{abstract}

MR Subject Classifications: 03E05, 04A20, 05A18, 05D10

Keywords: partition relations, Ramsey theory, real orders, transfinite ordinal numbers, triples

\section{A brief introduction}

In [3, Theorem 31, pp. 447-457], P. Erdős and R. Rado proved the theorem cited in the abstract, namely that if $X$ is an uncountable linear order into which neither $\omega_{1}$ nor $\omega_{1}^{*}$ embeds, then $X \rightarrow(\alpha, 4)^{3}$ for every ordinal $\alpha<\omega+\omega$. The proof they provided was quite complicated and difficult to follow. We thought it might be helpful to exhibit a simpler, more elementary proof.

\section{Notation and definitions}

We use standard set-theoretic notation as used in, for example, [4], [5], and [7].

An order is a set $X$ together with an ordering, a binary relation $<$ on $X$ which is transitive (if $x<y$ and $y<z$, then $x<z$ ) and irreflexive (never is $x<x$ ). If the 
ordering is trichotic (if always either $x<y, y<x$, or $x=y$ ), then the order is a linear order. For any order $X$ (with ordering $<$ ), the inversion of $X$ is the order $X^{*}$, with underlying set $X$ and ordering $<^{*}$ which is defined by putting $x<^{*} y$ if and only if $y<x$. For example, $\mathbb{R}^{*} \cong \mathbb{R}$, while $\omega^{*}$ is isomorphic to the negative integers with their usual ordering.

It is traditional when defining and describing orders to omit explicit mention of their orderings whenever possible, leaving them to be inferred from context or usage. We will continue this tradition, as it greatly simplifies notation and seldom seems to leads to confusion.

For any two orders $X$ and $Y$, we let $[X]^{Y}$ be the collection of all suborders of $X$ (subsets of $X$ together with the natural restrictions of its ordering) which are order isomorphic to $Y$. That is,

$$
[X]^{Y}=\{Z \subseteq X \mid Z \cong Y\}
$$

For example, $[\mathbb{R}]^{\omega}$ is the collection of all strictly increasing infinite sequences of real numbers, while $[\mathbb{R}]^{\mathbb{Q}}$ is the collection of all densely ordered sets of real numbers with neither maximal nor minimal element. Most importantly, for any order $X$ and any natural number $n$, we have that $[X]^{n}$ is the collection of all $n$-element chains of $X$;

$$
[X]^{n}=\left\{\left\{x_{0}, \ldots, x_{n-1}\right\} \mid x_{0}, \ldots, x_{n-1} \in X \wedge x_{0}<\cdots<x_{n-1}\right\} .
$$

And more generally, for two finite sequences of orders $X_{0}, \ldots, X_{m-1}$ and $Y_{0}, \ldots, Y_{m-1}$, we define (after P. Erdős and R. Rado)

$$
\left[X_{0}, \ldots, X_{m-1}\right]^{Y_{0}, \ldots, Y_{m-1}}=\left\{Z_{0} \cup \cdots \cup Z_{m-1} \mid Z_{0} \in\left[X_{0}\right]^{Y_{0}} \wedge \ldots \wedge Z_{m-1} \in\left[X_{m-1}\right]^{Y_{m-1}}\right\}
$$

the most important consequence of which is the fact that $[X, Y]^{1,2}$ is just the set of triples $\left\{x_{0}, y_{0}, y_{1}\right\}$ where $x_{0} \in X$ and $y_{0}, y_{1} \in Y$ with $y_{0}<y_{1}$.

We are interested in the combinatorics of orders, and most especially in their Ramsey theory, first described and investigated by P. Erdös and R. Rado in [3]. In its most straightforward form, Ramsey theory is the theory of the ordinary partition relation: Let $X$ be an order, $\mu$ be an ordinal, and each $Y_{i}$ for $i<\mu$ be an order. Then the partition relation $X \rightarrow\left(Y_{i}\right)_{i<\mu}^{n}$ holds if and only if for every partition $f:[X]^{n} \rightarrow \mu$ there are an index $i<\mu$ and a suborder $Z \in[X]^{Y_{i}}$ such that $f^{\text {" }}[Z]^{n}=\{i\}$. (In general, if $f$ is a function and $A$ is a set, then by $f$ " $A$ we mean the image of $A$ under $f$. That is, $f$ " $A=\{f(a) \mid a \in A\}$.) The failure of a partition relation is indicated by replacing

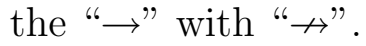

There are a few variations on this notation given in [3, Section 2, pp. 428-431], three of which we will need here. The first variation is concerned with the possibility that all of the orders $Y_{i}$ for $i<\mu$ are identical: The partition relation $X \rightarrow(Y)_{\mu}^{n}$ holds if for every partition $f:[X]^{n} \rightarrow \mu$ there are an index $i<\mu$ and a suborder $Z \in[X]^{Y}$ such that $f^{\text {" }}[Z]^{n}=\{i\}$. The second deals with the possibility that the number of classes in the partition is finite: The partition relation $X \rightarrow\left(Y_{0}, \ldots, Y_{m-1}\right)^{n}$ holds if 
for every partition $f:[X]^{n} \rightarrow\{0, \ldots, m-1\}$ there is an index $i<m$ and a suborder $Z \in[X]^{Y_{i}}$ such that $f^{"}[Z]^{n}=\{i\}$. The third variation allows for the possibility that all but one of the orders $Y_{i}$ for $i<\mu$ are identical: The partition relation $X \rightarrow\left((Y)_{\mu}, Z\right)^{n}$ holds if for every partition $f:[X]^{n} \rightarrow \mu+1$ either there are an index $i<\mu$ and a suborder $U \in[X]^{Y}$ such that $f^{"}[U]^{n}=\{i\}$ or there is a suborder $V \in[X]^{Z}$ such that $f^{"}[V]^{n}=\{\mu\}$. Examples of each of these variations appear in the results below.

An order is anti-well-founded if it contains no strictly increasing infinite sequences. That is, $X$ is anti-well-founded if and only if $[X]^{\omega}=\emptyset$. The character of an order is the minimum cardinal number of anti-well-founded suborders into which it can be decomposed. For example, the character of the first uncountable ordinal $\omega_{1}$ is $\omega_{1}$ (as every anti-well-founded suborder of $\omega_{1}$ is finite), while the character of the real line $\mathbb{R}$ is $2^{\omega}$ (as every anti-well-founded suborder of $\mathbb{R}$ is countable).

An order has countable character if it can be decomposed into countably many anti-well-founded suborders. Since every order can be decomposed into some number of anti-well-founded suborders (singletons, if need be), if an order does not have countable character, then it must have uncountable character. ${ }^{1}$ We remark that an order $X$ has countable character if and only if the negative partition relation $X \nrightarrow(\omega)_{\omega}^{1}$ holds. It follows that an order $X$ has uncountable character if and only if the positive partition relation $X \rightarrow(\omega)_{\omega}^{1}$ holds. It is a triviality that all countable orders and all anti-wellfounded orders have countable character.

A real order is a linear order with uncountable character into which $\omega_{1}$ does not embed. It is not difficult to see that the real line $\mathbb{R}$ is a real order (which explains the moniker "real"), as is any other uncountable linear order into which neither $\omega_{1}$ nor $\omega_{1}^{*}$ embeds. All real orders do not, however, fall into this latter class, as J. Baumgartner demonstrated in [2, Corollary 3.6, p. 194].

\section{A short proof}

The following theorem first appeared in [3, Theorem 31, pp. 447-457]. (Actually, this is not quite true. The theorem there was claimed only for uncountable orders into which neither $\omega_{1}$ nor $\omega_{1}^{*}$ embeds, rather than for all real orders; but the proof given there could be modified to yield this slightly stronger result.) The proof given below first appeared in [6, Section 5, pp. 32-34]. (Actually, this is not quite true, either. The proof given there was in spirit the one given below, but the result was again claimed only for a restricted class of real orders, namely those which cannot be decomposed into countably many scattered suborders. An order is scattered if it has no densely

\footnotetext{
${ }^{1}$ We feel obligated to note that the notions of having countable character and having uncountable character are both unique to this article; they are usually rendered as being special and being non-special, respectively. We believe, however, that these latter phrases are neither accurate nor illustrative, and hope that more useful alternatives can be found, perhaps the ones we suggest here or perhaps some others. With that said, we also wish to note that there is currently no term in common use for the notion of character defined above.
} 
ordered suborders.)

Theorem 1 (P. Erdös-R. Rado). If $X$ is a real order, then $X \rightarrow(\omega+m, 4)^{3}$ for every natural number $m$.

We will use the following "well known" facts in our proof of Theorem 1.

Fact 1 (P. Erdös-R. Rado). Every real order $X$ contains suborders $Y$ and $W$ such that

1. $Y$ is also a real order,

2. $W$ is (order) isomorphic to the ordinal $\omega^{2}$, and

3. $Y<W$ (i.e., $y<w$ for every $y \in Y$ and $w \in W$ ).

Fact 2 (F. Ramsey, P. Erdős-G. Szekeres). For each natural number $m$ there is a natural number $n$ such that $n \rightarrow(m, 4)^{3}$. Also, $\omega \rightarrow(\omega, 4)^{3}$.

Fact 3 (P. Erdös-R. Rado, E. Specker). The relation $\omega^{2} \rightarrow(n, \omega+m)^{2}$ holds for any two natural numbers $m$ and $n$.

Fact 4 (J. Baumgartner-A. Hajnal). For any two natural numbers $m$ and $n$, if $Z$ is a real order, then $Z \rightarrow\left((\omega+m)_{n}, \omega\right)^{2}$.

In each case a much stronger statement is true; for details we refer the interested reader to [3, Lemma 1, pp. 446-447], [3, Theorem 1, p. 431], [3, Theorem 23, pp. 439-440], and [1, Theorem 1, pp. 194-195], respectively. Evidently, all but the last fact were known to Erdös and Rado when they wrote [3].

Proof of Theorem 1. Let $X$ be a real order. Let a partition $f:[X]^{3} \rightarrow\{0,1\}$ be given. Fix a natural number $m$. We will show that either

(a) there is $A \in[X]^{\omega+m}$ with $f^{*}[A]^{3}=\{0\}$, or

(b) there is $B \in[X]^{4}$ with $f^{\text {" }}[B]^{3}=\{1\}$.

The claim below will be our most useful tool in this effort.

Claim. Suppose $x \in X$ and $A \in[X \backslash\{x\}]^{\omega+m}$ are such that $f^{\text {" }}[\{x\}, A]^{1,2}=\{1\}$. Then either (a) or (b) holds.

Proof of Claim. Clearly, if $f^{"}[A]^{3}=\{0\}$, then (a) holds. But what if $f "[A]^{3} \neq\{0\}$ ? Then there must be a triple $\left\{a_{0}, a_{1}, a_{2}\right\} \in[A]^{3}$ such that $f\left\{a_{0}, a_{1}, a_{2}\right\}=1$. Let $B=$ $\left\{x, a_{0}, a_{1}, a_{2}\right\}$. It is then easy to check that $f^{\prime \prime}[B]^{3}=\{1\}$, and hence that (b) holds. 
Using Fact 1, find $Y, W \subseteq X$, such that $Y$ is a real order, $W$ has order type $\omega^{2}$, and $Y<W$. For the remainder of the proof, we will focus our attention on the order $Y \cup W$ and the partition $f \uparrow[Y \cup W]^{3}:[Y \cup W]^{3} \rightarrow\{0,1\}$. It is important to keep in mind that $[Y \cup W]^{3}=[Y]^{3} \cup[Y, W]^{2,1} \cup[Y, W]^{1,2} \cup[W]^{3}$.

Using Fact 2, find a natural number $n$ such that $n \rightarrow(m, 4)^{3}$. For each $y \in Y$, define the partition $f_{y}:[W]^{2} \rightarrow\{0,1\}$ in the following way. For each pair of distinct elements $w_{0}$ and $w_{1}$ of $W$, put

$$
f_{y}\left\{w_{0}, w_{1}\right\}=f\left\{y, w_{0}, w_{1}\right\} .
$$

For each $y \in Y$, by Fact 3, either

(c) there is $A_{y} \in[W]^{\omega+m}$ with $f_{y}$ " $\left[A_{y}\right]^{2}=\{1\}$, or

(d) there is $D_{y} \in[W]^{n}$ with $f_{y} "\left[D_{y}\right]^{2}=\{0\}$.

If (c) holds for some $y \in Y$, then by the claim either (a) or (b) holds, and we are done. We may therefore assume (without loss of generality) that (d) holds for each $y \in Y$. That is, we may assume that for each $y \in Y$ there is an $n$-element subset $D_{y}$ of $W$ such that $f$ " $\left[\{y\}, D_{y}\right]^{1,2}=\{0\}$. Because $Y$ is a real order and $\left|[W]^{n}\right|=\omega$, there must be a real order $Z \subseteq Y$ and a particular $n$-element set $D=\left\{d_{0}, \ldots, d_{n-1}\right\} \subseteq W$ such that $D=D_{y}$ for every $y \in Z$. In particular, we note that

(d') $f$ " $[Z, D]^{1,2}=\{0\}$.

Define a partition $f_{D}:[Z]^{2} \rightarrow\{0, \ldots, n-1, n\}$ as follows. For each pair of distinct elements $z_{0}$ and $z_{1}$ of $Z$, put

$$
f_{D}\left\{z_{0}, z_{1}\right\}= \begin{cases}0 & \text { if } f\left\{z_{0}, z_{1}, d_{0}\right\}=1 \\ \vdots & \vdots \\ n-1 & \text { if } f\left\{z_{0}, z_{1}, d_{n-1}\right\}=1, \text { or } \\ n & \text { if } f\left\{z_{0}, z_{1}, d\right\}=0 \text { for each } d \in D .\end{cases}
$$

By Fact 4, either

(e) there are $i<n$ and $A \in[Z]^{\omega+m}$ such that $f_{D}$ "[A $]^{2}=\{i\}$ (and hence with $\left.f^{\prime \prime}\left[A,\left\{d_{i}\right\}\right]^{2,1}=\{1\}\right)$, or

(f) there is $C \in[Z]^{\omega}$ such that $f_{D} "[C]^{2}=\{n\}$ (and hence with $f^{*}[C, D]^{2,1}=\{0\}$ ).

If (e) holds, then by the claim, either (a) or (b) holds, and we are done. We may therefore assume (once again without loss of generality) that (f) holds. either

Consider the partition $f \uparrow[C]^{3}:[C]^{3} \rightarrow\{0,1\}$. Because $C \rightarrow(\omega, 4)^{3}$ (by Fact 2),

(g) there is $B \in[C]^{4}$ with $f^{*}[B]^{3}=\{1\}$, or 
(h) there is $E \in[C]^{\omega}$ with $f^{\text {" }}[E]^{3}=\{0\}$.

If (g) holds, then (b) follows, and we are done. We may therefore assume that (h) holds. Similarly, because $D \rightarrow(m, 4$ ) (by our choice of $n$ ), either

(i) there is $B \in[D]^{4}$ with $f^{\prime}[B]^{3}=\{1\}$, or

(j) there is $F \in[D]^{m}$ with $f^{\prime}[F]^{3}=\{0\}$.

As before, if (i) holds, then (b) follows, and we are done. We may therefore assume that (j) holds.

Finally, let $A=E \cup F$. Note that $A \in[X]^{\omega+m}$, since $E \in[X]^{\omega}, F \in[X]^{m}$, and $E<F$. Note also that $f "[E, F]^{1,2}=\{0\}$ by (d'); $f^{"}[E, F]^{2,1}=\{0\}$ by (f); $f^{"}[E]^{3}=\{0\}$ by $(\mathrm{h})$; and $f^{\text {" }}[F]^{3}=\{0\}$ by $(\mathrm{j})$. All of these together imply that $f^{\text {" }}[A]^{3}=\{0\}$. Thus (a) holds, and we are done.

\section{In conclusion}

We wish to consider the possibilities for improvement on Theorem 1. We know of two negative results which place direct limitations on such improvements, Theorems 2 and 3 below. (Incidentally, Theorem 2 provides an answer to a question of E. C. Milner and K. Prikry in [8, Section 1, p. 489].)

Theorem 2. Let $X$ be an order and $\kappa$ be an infinite cardinal. If $X$ has character no greater than $2^{\kappa}$, that is, if $X \nrightarrow(\omega){ }_{2}^{1}$, then $X \nrightarrow(\kappa+2, \omega)^{3}$. In particular, if $X$ is an order with character no greater than the cardinality of the continuum, that is, if $X \nrightarrow(\omega)_{2}^{1}$, then $X \nrightarrow(\omega+2, \omega)^{3}$.

Proof. Suppose $e:[X]^{1} \rightarrow{ }^{\kappa} 2$ witnesses that $X \nrightarrow(\omega){ }_{2^{\kappa}}^{1}$. Thus for no set $B \in[X]^{\omega}$ is $e$ constant on $[B]^{1}$. Consequently, for any set $B \in[X]^{\omega}$ there is a set $C \in[B]^{\omega}$ such that $e$ is one-to-one on $[C]^{1}$.

Define a partition of pairs $f:[X]^{2} \rightarrow \kappa+1$ as follows. For each pair $x, y \in X$ with $x<y$, let

$$
f\{x, y\}=\delta(e\{x\}, e\{y\})
$$

where $\delta(s, t)=\min (\{\xi<\kappa \mid s(\xi) \neq t(\xi)\} \cup\{\kappa\})$ for each pair of sequences $s$ and $t$ in ${ }^{\kappa} 2$. Next, define a partition of triples $g:[X]^{3} \rightarrow\{0,1\}$ as follows. For each triple $x, y, z \in X$ with $x<y<z$, let

$$
g\{x, y, z\}= \begin{cases}0 & \text { if } e \text { is one-to-one on }[\{x, y, z\}]^{1} \text { and } f\{x, y\}<f\{y, z\} \\ 1 & \text { if } e \text { is not one-to-one on }[\{x, y, z\}]^{1} \text { or } f\{x, y\} \geq f\{y, z\}\end{cases}
$$

This partition does the trick: 
Claim. There is no set $A \in[X]^{\kappa+2}$ with $g^{\text {" }}[A]^{3}=\{0\}$.

Assume to the contrary that there is $A \in[X]^{\kappa+2}$ with $g^{\prime \prime}[A]^{3}=\{0\}$. Note that $e$ is necessarily one-to-one on $[A]^{1}$, by the definition of $g$. Enumerate $A$ in increasing order as

$$
A=\left\{x_{0}, x_{1}, \ldots, x_{\alpha}, \ldots, x_{\kappa}, x_{\kappa+1}\right\} .
$$

Let $\xi=f\left\{x_{\kappa}, x_{\kappa+1}\right\}$. Since $e\left\{x_{\kappa}\right\} \neq e\left\{x_{\kappa+1}\right\}$, it must be that $\xi<\kappa$. Next, we note that for any triple $s, t, u \in{ }^{\kappa} 2$, if $\delta(s, t)<\delta(t, u)$, then $\delta(s, u)=\delta(s, t)$. Thus, for every pair $\alpha<\beta<\kappa$ we have that $f\left\{x_{\alpha}, x_{\kappa}\right\}=f\left\{x_{\alpha}, x_{\beta}\right\}<f\left\{x_{\beta}, x_{\kappa}\right\}$. But also, $f\left\{x_{\alpha}, x_{\kappa}\right\}<f\left\{x_{\kappa}, x_{\kappa+1}\right\}=\xi$ for each $\alpha<\kappa$. But this is absurd; if we define $h: \kappa \rightarrow \kappa$ by letting $h(\alpha)=f\left\{x_{\alpha}, x_{\kappa}\right\}$ for each $\alpha<\kappa$, then the preceding remarks tell us that $h(\alpha)<h(\beta)$ for each pair $\alpha<\beta<\kappa$ and yet $h " \kappa \subseteq \xi<\kappa$, which is impossible. Thus no such set $A \in[X]^{\kappa+2}$ exists.

Claim. There is no set $B \in[X]^{\omega}$ with $g^{"}[B]^{3}=\{1\}$.

Assume to the contrary that there is $B \in[X]^{\omega}$ with $g^{\prime \prime}[B]^{3}=\{1\}$. By the remarks at the beginning of the proof, we may assume without loss of generality that $e$ is oneto-one on $[B]^{1}$. Consider a new partition $h:[B]^{3} \rightarrow\{0,1\}$ defined as follows. For each triple $x, y, z \in B$ with $x<y<z$, let

$$
h\{x, y, z\}= \begin{cases}0 & \text { if } f\{x, y\}>f\{y, z\} \\ 1 & \text { if } f\{x, y\}=f\{y, z\} .\end{cases}
$$

Since $g^{"}[B]^{3}=\{1\}$, this partition is well-defined. But also, since $\omega \rightarrow(\omega, 4)^{3}$, either

(a) there is $C \in[B]^{\omega}$ such that $h^{"}[C]^{3}=\{0\}$, or

(b) there is $D \in[B]^{4}$ such that $h^{\prime \prime}[D]^{3}=\{1\}$.

If (a) is true, then there would be an infinite descending sequence of ordinals, which is absurd. If (b) is true, then there would be three distinct sequences in ${ }^{\kappa} 2$, each pair of which first differed at the same point, which is also absurd. Thus no such set $B \in[X]^{\omega}$ exists.

Theorem 3. Let $X$ be an order and $\kappa$ be an infinite cardinal. If $X \nrightarrow(\mathrm{cf} \kappa)_{\kappa}^{1}$, then $X \nrightarrow(\kappa+1,4)^{3}$. In particular, if $X$ has countable character, then $X \nrightarrow(\omega+1,4)^{3}$.

Proof. Suppose the partition $e:[X]^{1} \rightarrow \kappa$ witnesses that $X \nrightarrow(\operatorname{cf} \kappa)_{\kappa}^{1}$. Thus for no set $C \in[X]^{\text {cf } \kappa}$ is $e$ constant on $[C]^{1}$. Consequently, for any set $A \in[X]^{\kappa}$ there is a set $B \in[A]^{\kappa}$ such that $e$ is one-to-one on $[B]^{1}$. The next claim goes this one step better.

Claim. For any set $A \in[X]^{\kappa}$ there is a subset $B \in[A]^{\kappa}$ such thate is strictly increasing on $[B]^{1}$. (That is, such that $e\{x\}<e\{y\}$ for any pair $x, y \in B$ with $x<y$.) 
Proof. It is a well-known theorem of B. Dushnik, P. Erdős, and E. W. Miller (see, for example, [3, Theorem 44, pp. 475-476]) that $\kappa \rightarrow(\kappa, \omega)^{2}$ for any infinite cardinal $\kappa$. If we define a partition $f:[A]^{2} \rightarrow\{0,1\}$ by letting

$$
f\{x, y\}= \begin{cases}0 & \text { if } e\{x\} \leq e\{y\} \\ 1 & \text { if } e\{x\}>e\{y\}\end{cases}
$$

for each pair $x, y \in A$ with $x<y$, then this tells us that either

(c) there is a set $C \in[A]^{\kappa}$ such that $f^{\prime \prime}[C]^{2}=\{0\}$, or

(d) there is a set $D \in[B]^{\omega}$ such that $f^{\prime \prime}[D]^{2}=\{1\}$.

If (d) is true, then $e^{\text {" }[D]^{1}}$ would constitute an infinite strictly decreasing sequence of ordinals, which is absurd. Thus (c) must be true. As we mentioned above, there must be a set $B \in[C]^{\kappa}$ with $e$ one-to-one on $[B]^{1}$. Clearly, $e$ is strictly increasing on $[B]^{1}$.

Define a partition of triples $f:[X]^{3} \rightarrow\{0,1\}$ as follows. For a triple $x, y, z \in X$ with $x<y<z$, let

$$
f\{x, y, z\}= \begin{cases}0 & \text { if either } e\{x\} \geq e\{y\} \text { or } e\{y\} \leq e\{z\} \\ 1 & \text { if both } e\{x\}<e\{y\} \text { and } e\{y\}>e\{z\}\end{cases}
$$

We will show that neither

(a) is there $A \in[X]^{\kappa+1}$ such that $f "[A]^{3}=\{0\}$, nor

(b) is there $B \in[X]^{4}$ such that $f^{\text {" }}[B]^{3}=\{1\}$.

For suppose there is $A \in[X]^{\kappa+1}$ with $f^{"}[A]^{3}=\{0\}$. Enumerate $A$ in ascending order as $A=\left\{x_{0}, x_{1}, \ldots, x_{\alpha}, \ldots, x_{\kappa}\right\}$. By the claim, we may assume that $e$ is strictly increasing on $\left[A \backslash\left\{x_{\kappa}\right\}\right]^{1}$. Consider triples of the form $\left\{x_{\alpha}, x_{\beta}, x_{\kappa}\right\}$ for $\alpha<\beta<\kappa$; by the definition of $g$, because $e\left\{x_{\alpha}\right\}<e\left\{x_{\beta}\right\}$, it must be that $e\left\{x_{\beta}\right\} \leq e\left\{x_{\kappa}\right\}$ for each $\beta<\kappa$. But this is absurd, as $e^{"[}\left[A \backslash\left\{x_{\kappa}\right\}\right]^{1}$ is cofinal in $\kappa$ (because $e$ is strictly increasing on $[A]^{1}$ ). Thus no such set $A \in[X]^{\kappa+1}$ exists.

Suppose also that there is $B \in[X]^{4}$ with $f^{\text {" }}[B]^{3}=\{1\}$. Enumerate $B$ in increasing order as $\left\{y_{0}, y_{1}, y_{2}, y_{3}\right\}$. Because $g\left\{y_{0}, y_{1}, y_{2}\right\}=1$, it must be that $y_{1}>y_{2}$. Because $g\left\{y_{1}, y_{2}, y_{3}\right\}=1$, it must be that $y_{1}<y_{2}$. Clearly this is absurd; thus no such set $B \in[X]^{4}$ exists.

In the light of Theorems 2 and 3 , a positive resolution of the following question would be the best improvement of Theorem 1 possible.

Question 1. If $X$ is an order with uncountable character, then does $X \rightarrow(\alpha, n)^{3}$ for every countable ordinal $\alpha$ and every natural number $n$ ? 
Currently, the best results toward a resolution of this question are due to E. C. Milner and K. Prikry, who demonstrated in [9, Section 3, pp. 185-190] that $\omega_{1} \rightarrow(\omega+$ $\omega+1,4)^{3}$; and to the author, who was able to show in [6, Sections 4 and 5, pp. 35-52] that if $X$ is an order with uncountable character, then $X \rightarrow(\alpha, n)^{3}$ for any ordinal $\alpha<\omega+\omega$ and any natural number $n<\omega$. These two results make the final questions below the simplest open cases of Question 1.

Question 2. If $X$ is an order with uncountable character (into which $\omega_{1}$ does not embed), does then $X \rightarrow(\omega+\omega, 4)^{3}$ ? In particular, does $\mathbb{R} \rightarrow(\omega+\omega, 4)^{3}$ ?

Question 3. Does $\omega_{1} \rightarrow(\omega+\omega+2,4)^{3}$ ?

Question 4. Does $\omega_{1} \rightarrow(\omega+\omega, 5)^{3}$ ?

\section{References}

[1] J. Baumgartner and A. Hajnal. A proof (involving Martin's axiom) of a partition relation. Fund. Math., 78(3):193-203, 1973.

[2] James E. Baumgartner. A new class of order types. Ann. Math. Logic, 9(3):187-222, 1976.

[3] P. Erdös and R. Rado. A partition calculus in set theory. Bull. Amer. Math. Soc., 62:427-489, 1956.

[4] Paul Erdős, András Hajnal, Attila Máté, and Richard Rado. Combinatorial set theory: partition relations for cardinals. North-Holland Publishing Co., Amsterdam, 1984.

[5] T. Jech. Set Theory, volume 79 of Pure and Applied Mathematics. Academic Press, 1978.

[6] A. Jones. Some results in the partition calculus. PhD thesis, Dartmouth College, June 1999.

[7] Akihiro Kanamori. The higher infinite. Springer-Verlag, Berlin, 1994. Large cardinals in set theory from their beginnings.

[8] E. C. Milner and K. Prikry. A partition theorem for triples. Proc. Amer. Math. Soc., 97(3):488-494, 1986.

[9] E. C. Milner and K. Prikry. A partition relation for triples using a model of Todorčević. Discrete Math., 95(1-3):183-191, 1991. Directions in infinite graph theory and combinatorics (Cambridge, 1989). 